\title{
Evaluating a genetically encoded optical sensor of neural activity using electrophysiology in intact adult fruit flies
}

\author{
Vivek Jayaraman ${ }^{\dagger}$ and Gilles Laurent ${ }^{*}$ \\ Computation and Neural Systems Program, Division of Biology, California Institute of Technology, USA \\ Edited by: Rafael Yuste, Columbia University, New York City, USA \\ Reviewed by: Karen Zito, University of California Davis, Davis, United States of America \\ Rafael Yuste, Columbia University, New York City, USA
}

\begin{abstract}
Genetically encoded optical indicators hold the promise of enabling non-invasive monitoring of activity in identified neurons in behaving organisms. However, the interpretation of images of brain activity produced using such sensors is not straightforward. Several recent studies of sensory coding used G-CaMP 1.3-a calcium sensor-as an indicator of neural activity; some of these studies characterized the imaged neurons as having narrow tuning curves, a conclusion not always supported by parallel electrophysiological studies. To better understand the possible cause of these conflicting results, we performed simultaneous in vivo 2-photon imaging and electrophysiological recording of G-CaMP 1.3 expressing neurons in the antennal lobe (AL) of intact fruitflies. We find that G-CaMP has a relatively high threshold, that its signal often fails to capture spiking response kinetics, and that it can miss even high instantaneous rates of activity if those are not sustained. While G-CaMP can be misleading, it is clearly useful for the identification of promising neural targets: when electrical activity is well above the sensor's detection threshold, its signal is fairly well correlated with mean firing rate and G-CaMP does not appear to alter significantly the responses of neurons that express it. The methods we present should enable any genetically encoded sensor, activator, or silencer to be evaluated in an intact neural circuit in vivo in Drosophila.
\end{abstract}

Keywords: Drosophila, neural coding, olfaction, antennal lobe, 2-photon imaging, electrophysiology, genetically encoded calcium indicators, G-CaMP

\section{INTRODUCTION}

The fruit fly has been a mainstay of behavioral genetics for many decades (Benzer, 1967; Hotta and Benzer, 1970; Quinn et al., 1974). It has not, however, been a model system of choice for systems neuroscience. One reason was the lack of accessibility of its neurons for in vivo recordings. Recent developments show, however, that it is possible to record in vivo from fly brain neurons with intracellular electrophysiology (Wilson et al., 2004). This approach is direct and has high temporal resolution. Of all the electrophysiological recording methods available, whole-cell patch-clamp technique (Sakmann and Neher, 1984) may be the most appropriate for small neurons, such as of Drosophila (Wilson et al., 2004; Wilson and Laurent, 2005). Whole-cell patch-clamp, however, is invasive: it causes dialysis and often requires direct current injection to hold the neuron at a desired potential, when the neuron's normal resting potential is unknown. It is also limited in that only a few neurons at the most can be sampled simultaneously. Alternative techniques use optical reporters of voltage (Cohen et al., 1978; Cohen and Salzberg, 1978; Grinvald and Hildesheim, 2004; Taylor et al., 2003) or intracellular calcium (Grynkiewicz et al., 1985; Helmchen et al., 1996; Stosiek et al., 2003; Svoboda et al.,

\footnotetext{
* Correspondence: Gilles Laurent, Computation and Neural Systems Program, Division of Biology, California Institute of Technology, Pasadena, CA 91125, USA. e-mail: laurentg@caltech.edu; or Vivek Jayaraman, e-mail: vivek@janelia.hhmi.org

$\dagger$ Current address: Howard Hughes Medical Institute, Janelia Farm Research Campus, 19700 Helix Drive, Ashburn, VA 20147, USA.

Received: 10 Aug. 2007; paper pending published: 17 Sep. 2007; accepted: 15 0ct. 2007; published online: 2 Nov. 2007

Full citation: Frontiers in Neural Circuits (2007) 1:3 doi: 10.3389/neuro.04/003.2007 Copyright: () 2007 Jayaraman, Laurent. This is an open-access article subject to an exclusive license agreement between the authors and the Frontiers Research Foundation, which permits unrestricted use, distribution, and reproduction in any medium, provided the original authors and source are credited.
}

1997; Tank et al., 1988; Yuste and Katz, 1991). Some of these indicators are now genetically encoded (Miyawaki et al., 1997; Nakai et al., 2001; Persechini et al., 1997); using genetic techniques (Brand and Perrimon, 1993), specific cell groups can be targeted selectively (Fiala et al., 2002; $\mathrm{Ng}$ et al., 2002; Schroll et al., 2006; Wang et al., 2003), making optical approaches very powerful. Yet, these methods have their own intrinsic limitations. Among them is the interpretability of the signal they produce: natural concerns are about their temporal resolution, sensitivity, and correlation to electrical activity (Pologruto et al., 2004; Reiff et al., 2005). These characteristics may vary with cell type and expression level. In addition, because calcium indicators are modified calcium buffers, their presence in neurons could alter development or function. Thus, it seems important to evaluate the performance of these sensors in the cells of interest before using them as standalone indicators of neural activity.

Combining simultaneous loose-patch-clamp recordings and twophoton calcium imaging (Denk et al., 1990; Smetters et al., 1999), we performed such direct calibration experiments with G-CaMP-expressing projection neurons (PNs) in the fruit fly antennal lobe (AL). The AL is the first relay in the insect olfactory system and has been the subject of many recent investigations (Olsen et al., 2007; Shang et al., 2007; Wang et al., 2003; Wilson etal., 2004; Yu et al., 2004). Most Drosophila PNs each arborize in one glomerulus, where they receive direct afferent input from one olfactory sensory neuron (OSN) type. An issue that has received some attention recently concerns the tuning of PNs to odors: studies based on whole-cell patch-clamp recordings (Olsen et al., 2007; Wilson et al., 2004) report that most PNs respond broadly, and are more broadly tuned than their presynaptic OSNs. By contrast, a study based on two-photon imaging with G-CaMP found sparse PN responses to odors (Wang et al., 2003) suggesting a faithful transfer of representations from OSNs to PNs. Tuning width has consequences on coding efficiency, making this issue relevant 
to olfaction in particular and sensory systems in general. The simplest reason for this discrepancy is technical: one set of studies used wholecell patch-clamp recordings from intact flies, the other G-CaMP imaging from isolated fly heads. The present experiments, because they consist of electrophysiological recordings simultaneously with G-CaMP imaging in intact flies, should help resolve this issue directly.

\section{MATERIALS AND METHODS \\ Fly stocks}

Flies were reared on standard cornmeal agar medium. We used the Gal4/UAS-system (Brand and Perrimon, 1993) to direct the expression of the calcium sensor G-CaMP (Nakai et al., 2001) to specific cells. GH146Gal4 flies were a gift from L. Luo (Stanford University, Stanford, CA). UAS-GCaMP flies were a gift from R. Axel (Columbia University, New York, NY). NP3529-Gal4 flies were a gift from K. Ito (The University of Tokyo, Japan). All experimental animals were adult females, 1-2 days after eclosion.

\section{Fly preparation}

Adult flies were dissected using recently described methods (Wilson et al., 2004). Flies were anesthetized in a glass vial on ice just until movement stopped ( $\sim 15$ second) and then gently inserted into a hole in a piece of aluminum foil. Small drops of wax $\left(55^{\circ} \mathrm{C}\right)$ were used to suspend the fly in the hole, with the edge of foil defining a horizontal plane around the head and thorax, from the first antennal segment anteriorly to the scutellum posteriorly. The dorsal side of the foil was bathed in saline, while the ventral side (including antennae and maxillary palps) remained dry and accessible to odors. A window was cut in the dorsal head cuticle between the eyes, extending from the ocelli to the first antennal segment. Fat and air sacs dorsal and anterior to the brain were removed, and the perineural sheath was gently picked away from the ALs. In some preparations, brief exposure to a collagenase solution was used to weaken the sheath; activity in these brains was not noticeably different from brains desheathed mechanically. The proboscis was affixed with a small drop of wax to a strand of human hair to limit brain movement. To further limit brain movement, the pair of small muscles (muscle 16) lying between and ventral to the antennal nerves was also removed. Spontaneous leg movements were typically observed in this preparation for the duration of the recording (1.5-3 hour). The saline composition used in all experiments was (Wang et al., 2003): $108 \mathrm{mM} \mathrm{NaCl}, 5 \mathrm{mM} \mathrm{KCl}, 2 \mathrm{mM} \mathrm{CaCl}_{2}, 8.2 \mathrm{mM} \mathrm{MgCl}_{2}, 4 \mathrm{mM} \mathrm{NaHCO}_{3}$, $1 \mathrm{mM} \mathrm{NaH}_{2} \mathrm{PO}$, $5 \mathrm{mM}$ trehalose, $10 \mathrm{mM}$ sucrose, $5 \mathrm{mM}$ HEPES [pH 7.5, 265 m0sm].

\section{Odor delivery}

Odors (cis-3-hexen-1-ol (cis), benzaldehyde (ba), isoamyl acetate (ia), 1hexanol (hex), eugenol (eug), acetophenone (ace), citral (cit), cherry (che)) were delivered using a custom-made odor-delivery system and a Teflon nozzle (entry diameter $1 \mathrm{~cm}$, exit $\sim 0.1 \mathrm{~cm}$ ) directed towards the antennae. Odors were delivered in a constant stream of air (0.4-0.8 l/second) at final concentrations of ca. $0.5-50 \%$. The results reported are based on data obtained from 24 PNs in 22 flies. Not all odors could be tested in all animals and the number of trials of each odor presented varied from 1 to 6 , depending on the stability of the recording.

\section{Electrophysiology: Loose-patch recordings}

Patch-clamp electrodes (4-5 $\mathrm{M} \Omega$ ) were pulled on a Sutter Instruments (Novato, CA) P87 horizontal puller and pressure polished before filling with a solution containing $0.1 \mathrm{mM}$ sulforhodamine B (Molecular Probes, Eugene, $\mathrm{OR}$ ) in saline solution. PNs were approached using two-channel imaging (Margrie et al., 2003) on a two-photon microscope. Low resistance $(100-300 \mathrm{M} \Omega$ ) seals allowed spikes to be reliably detectable for up to 40 minute, although some useable recordings lasted only 5 minute before an increase in seal resistance or spontaneous break-in. Current data were acquired via an Axopatch-1D amplifier (Axon Instruments, Union
City, CA), a National Instruments A-D card (15 kHz sampling) and LabView software (National Instruments, Austin, TX).

\section{Electrophysiology: Spike detection}

To detect spikes in loose patch recordings, we used a combination of criteria that were kept constant for all traces recorded during an experiment. The current traces were first bandpass filtered $(10 \mathrm{~Hz}-1 \mathrm{kHz})$ and then boxcar filtered ( $\sim 1 \mathrm{~ms}$ window). We then passed the traces through an amplitude threshold that was held constant for all trials of a given PN. Negative peaks detected in this thresholded trace were considered candidate spikes and were subjected to two further tests. First, we applied a slope threshold by computing the deflection from the negative peak to a point on the trace 2 ms later. This threshold-typically twice the amplitude threshold in magnitude, and always held constant for all trials of a given $\mathrm{PN}$-was typically sufficient for clean spike detection (evaluated both manually and by clustering). However, for a few noisier recordings, we performed another test by using a template spike. The template spike was chosen by eye. Thresholding the dot product of this waveform with all spike candidates was sufficient to resolve remaining ambiguities.

\section{Imaging}

A two-photon microscope based on a galvanometric-mirrors-scan-system (Denk et al., 1990) and proximal detection (Radiance, 2001; BioRad, Hercule, CA) coupled to an upright microscope (BX51-Wl; Olympus, Japan) was used to image calcium signals from PNs. A mode-locked Ti: sapphire laser (Tsunami-10 W pump; Spectra Physics, Mountain View, CA) tuned to $920-935 \mathrm{~nm}$ was used as excitation light. A whole-field DOT-system used in reflection allowed precise positioning of the brain and electrode, mounted on XY-translation stages. Non-prechirped excitation pulses were focused onto labeled PNs using a $40 \times 0.75$ NA or $63 \times 0.90$ NA water immersion lens (Leica Microsystems, Germany). The epi-collected fluorescence was bandpass filtered (Green: HQ515-30, Red: HQ620-100, Chroma Technology, Rockingham, VT) and detected with two photomultiplier tubes (bi-alkali photo cathode; Electron Tubes, UK), one for each channel (red to visualize the electrode and green for the cell). Application of up to $50 \mathrm{~mW}$ into the sample was sufficient to image somata located at a depth of up to $50 \mu \mathrm{m}$. To minimize photodamage, excitation power was adjusted with the depth of the focal plane using a liquid crystal based attenuator (NewPort, Irvine, CA) and kept to a minimum for sufficient signal-to-noise $(\mathrm{S} / \mathrm{N})$ ratio. No apparent changes in PN morphology, calcium signal or electrical properties resulted from laser illumination. Twenty-four second-long line scans (512 pixel lines at 500 or $600 \mathrm{~Hz}$ ) were performed across the width of individual somata in their equatorial plane, and acquired with 16-bit resolution. Imaging and physiological data acquisitions were synchronized with a precision $<1 \mathrm{~ms}$ from a common pulsed command.

\section{Data analysis}

Electrophysiological and line-scan data were combined and analyzed offline using LabView (National Instruments, Austin, TX) and MATLAB (The MathWorks Inc., Natick MA) software. For line-scan data, we excluded the first 2 second of data, and then performed a linear bleach correction using a scale factor calculated using fluorescence levels for 1 second before the odor stimulus was delivered ( $4-5$ second) and 1 second before the end of the line-scan (23-24 second). Consistent with previous results (Reiff et al., 2005), photobleaching appeared to increase with increases in fluorescence intensity, explaining the dips seen in G-CaMP signal immediately after strong responses. Data were box-car filtered $(20 \mathrm{~ms}$ for all comparisons with electrophysiology and in Figure 4; $40 \mathrm{~ms}$ otherwise) before further analysis. In all cases fluorescence changes were calculated relative to baseline fluorescence levels as determined by averaging over 2 second just before odor presentation. To compute first detectable deviations from baselines, we used a threshold of $2.5 \mathrm{SDs}$. Times reported are when imaging signal first rose above this threshold.

2 


\section{RESULTS}

Two-photon targeted electrophysiological recordings

GCaMP-Gal4, UAS-GCaMPflies (Wang et al., 2003) were used for a series of two-photon targeted electrophysiological recordings from PNs in the fly AL. Approximately half of the PN population is targeted by this line: G-CaMP-expressing PNs showed strong baseline fluorescence, and PNs showed strong and selective changes in fluorescence in response to odors (Figure 1A).

For electrophysiological recordings, we used the loose-patch technique. This minimally invasive technique allowed us to gently seal onto PNs and observe their spiking activity without breaking in $(n=8$; seven flies). Cells were targeted visually using two-channel imaging (Figure 1B, see Materials and Methods). Occasionally, we were able to break into the cell at the end of an experiment and confirm cell identity. However, this was usually not possible, and we could only use the two-channel images of the patch pipette near the cell for confirmation of pipette-soma apposition. The red dye in the pipette was occasionally blown out gently to allow visualization of otherwise non-fluorescent - and therefore invisible - neuropil and non-targeted cells in the neighborhood of the neuron of interest.

Loose-patch recordings, although noisy (because of the low-resistance seals), were fairly stable for up to 40 minute. PNs responded reliably
A

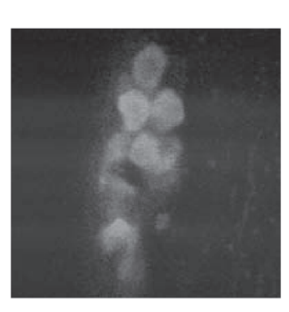
$10 \mu \mathrm{m}$
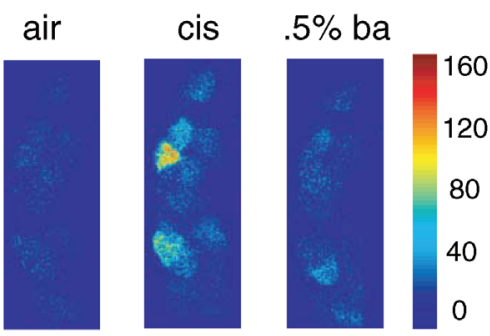

$\Delta \mathrm{F}$
B
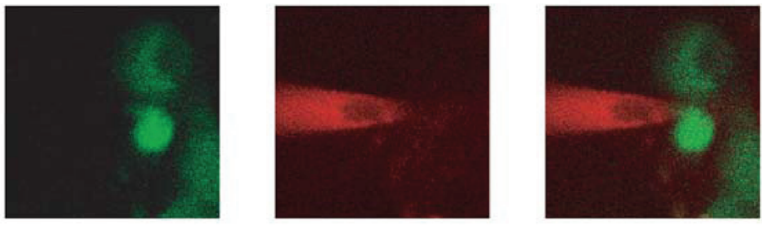

$5 \mu \mathrm{m}$

C
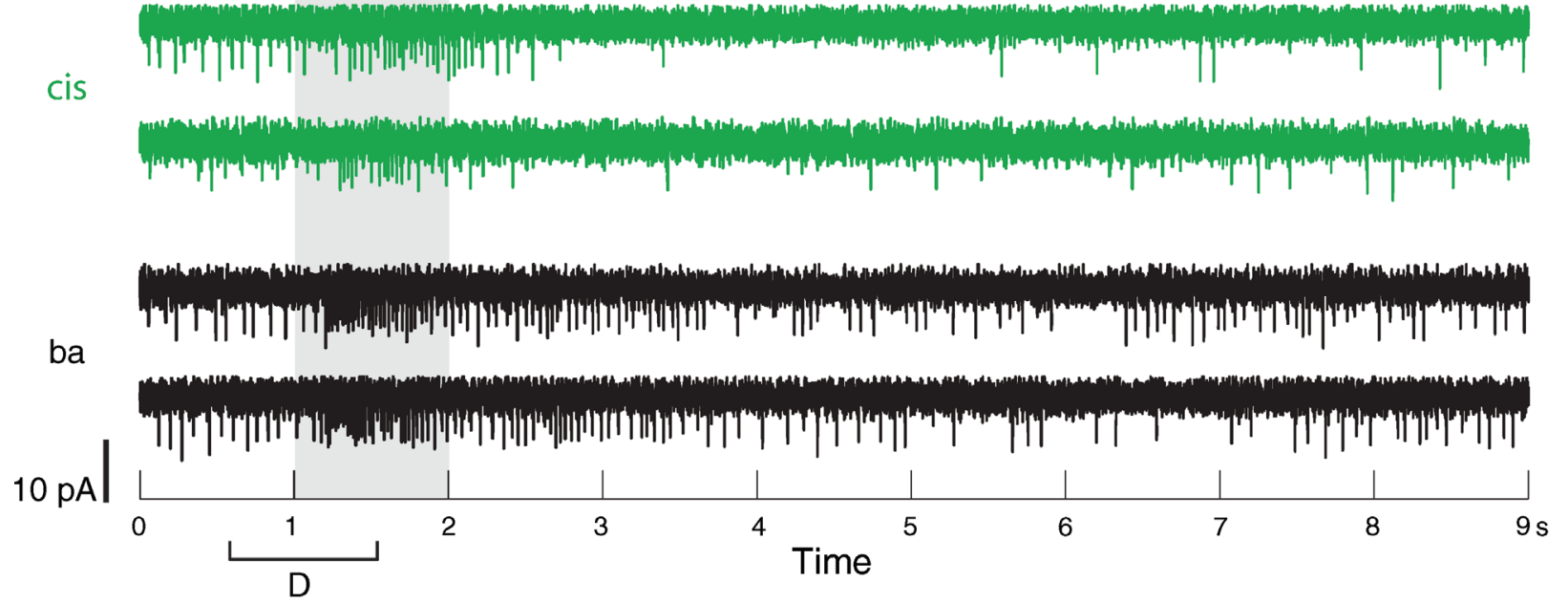

D

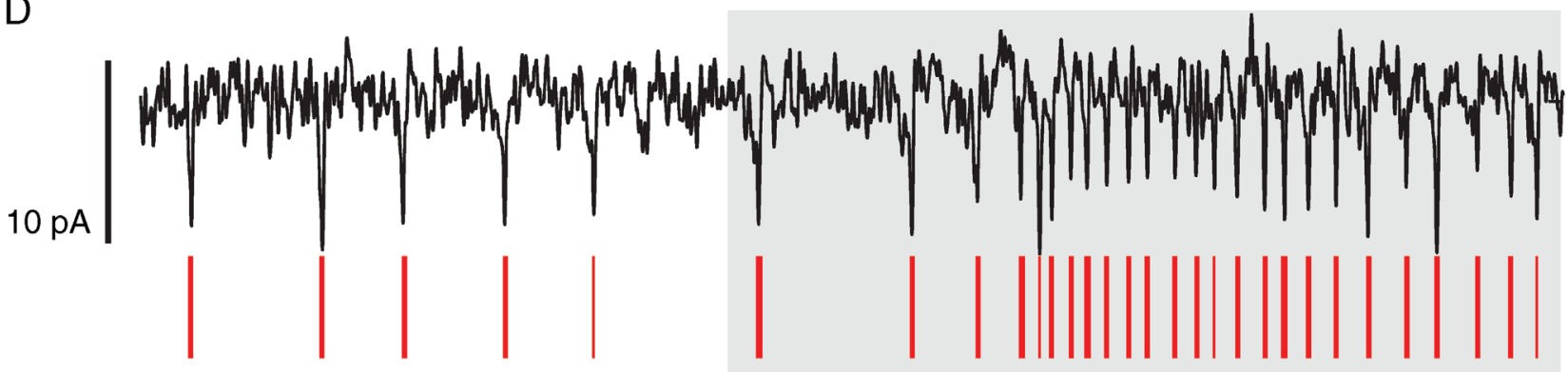

$100 \mathrm{~ms}$

Figure 1. Loose-patch recording from identified PNs in GH146-Gal4, UAS-GCaMP flies. (A) Baseline levels of fluorescence (left) of G-CaMP-expressing dorsal PNs, and absolute changes in (8-bit) fluorescence intensity levels in response to air and two odor presentations. (B) Two-channel imaging showing (from left) images in the green, and red channels; image on right is an image created from merging the two channels. Images verify that cell was not broken into during recording. (C) Current traces from the PN above. Shown are responses to two different odor presentations (two trials each) (gray bar). (D) Spikes, although small, can be separated from the noise by a combination of amplitude and fast deflection (see Materials and Methods). Red lines indicate spikes detected automatically. 
with excitation and inhibition (visible as a reduction in instantaneous rate relative to baseline) to different odors (Figure $1 \mathrm{C}$ ). Spike adaptation during bursts of activity meant a temporary decrease in $\mathrm{S} / \mathrm{N}$, but spikes were easily detected by their amplitude and slope even in periods of fast firing (Figure 1D).

\section{Effect of G-CaMP on normal neuronal function}

The introduction of calcium sensors can impact a cell's calcium dynamics (Yasuda et al., 2004). If the sensor is present from the birth of the neuron, it might possibly change its physiology and affect network activity in the targeted population. To investigate this possibility in G-CaMP-positive cells, we performed experiments on identified PNs that project to and receive afferent input from the DL1 glomerulus; these PNs are selectively labeled in the fly line NP3529-Gal4 (Tanaka et al., 2004). After crossing with a UAS-eGFP line, we obtained flies with enhanced-GFP-labeled DL1 PNs. These eGFP flies were used as controls (cytosolic GFP is not considered to have deleterious effects on cell physiology (Su and 0'Dowd, 2003)). Comparisons of the physiology of G-CaMP-expressing DL1 PNs ( $n=6,5$ flies) and GFP-expressing DL1 PNs ( $n=5,5$ flies) revealed no major differences (Figures 2A-2G). Spontaneous firing was limited in both cases, with mean baseline firing rates of $1.33 \pm 2.32 \mathrm{sp} /$ second for the G-CaMP PNs and $0.33 \pm 0.5 \mathrm{sp} / \mathrm{second}$ for the GFP PNs (difference of means not significant, two-sample $t$-test, $p=0.3556$ ). The PNs also showed similar —albeit not identical — odor response profiles. For example, responses to benzaldehyde (ba) consistently featured two peaks in instantaneous firing rate, one each immediately after odor onset and offset (Figures 2A and $2 \mathrm{C}$ ). The same was true, to a lesser extent, for isoamyl acetate (ia) (Figures 2B and 2D). Although the mean offset responses for the GFP PNs was different from that seen in G-CaMP PNs (Figures $2 \mathrm{C}$ and 2D), they were within a single SD of each other. Responses to acetophenone (ace) showed a similar early increase in firing followed by sustained firing at lower rates for over 20 second in both lines (Figure $\mathbf{2 G}$ ). Inter-individual response variability in PNs of one type (GFP or G-CaMP) was fairly high (see SD of peri-stimulus time histograms (PSTHs) in Figure 2) relative to differences between the two types (visible in mean traces in Figure 2). We performed statistical comparisons of mean rate during response (1-second and 3-second window immediately after odor onset, the latter capturing most of the period of above-baseline G-CaMP signal in response to odor presentation) for the five odors tested on at least 3 PNs of each type. We found no significant differences between the two groups (two-sample $t$ test: $p \gg 0.05$ for all comparisons except ia, which had a $p$-value of 0.09 for a comparison using a 3-second window for mean rate computation), nor for any intermediate time windows we tested. In conclusion, present indications are that G-CaMP, at the expression level tested, does not have any more significant effects on PN response patterns than GFP.

\section{Calibration of G-CaMP 1.3 using simultaneous electrophysiology and imaging}

To test the effectiveness of G-CaMP as a reporter of neural activity, we combined electrophysiological recording and simultaneous G-CaMP imaging of individual PN responses to odors. We used GH146-Gal4, UAS-GCaMP flies for all these experiments with four copies of G-CaMP (Wang et al., 2003). In all these experiments ( $n=8$, seven flies), baseline fluorescence was high, and changes in fluorescence were observed to at least one of the odors tested. This excludes the possibility of problems with the acquisition of the imaging signal.

At the start of each experiment, we identified an "image-positive" PN, that is, one that displayed a detectable G-CaMP response to at least one of the odors tested. We then performed line-scans of the equator of the soma at low laser intensity (sufficient for visible baseline fluorescence) and targeted the same soma with an electrode for loose-patch recording (Figure 3A). The raw fluorescence signal (Figures 3B and 3C) was boxcar filtered (Figure 3D) before comparisons were made with electrophysiological responses (Figures 3D and 3E). In this example, we found that G-CaMP was able to detect responses to all three odors, albeit with some lag ( $>500 \mathrm{~ms}$, measured as the delay between times of firing rate increases of 2.5 SDs above baseline and fluorescence changes of 2.5 SDs over baseline) and with a slow decay (G-CaMP signal returned to baseline up to 5 second later than firing rate did).

In a different PN with clear spiking responses to three odors (Figure 3F), G-CaMP signal only reflected a strong response to one odor (cherry). With the odor ia, the evoked spiking response had a peak instantaneous rate (measured in 50-ms windows) of 40 spikes/second; this response went undetected with G-CaMP (Figure $3 \mathrm{G}$ ). This was a consistent trend across all simultaneous recordings, indicating that although G-CaMP is sufficiently sensitive to detect high rates of sustained activity, the sensor has a high threshold and slow kinetics at this expression level. We next measured the correlation between detected G-CaMP signal (defined relative to baseline noise), and spiking activity. This correlation (mean response rate vs. mean fluorescence change) was 0.61 (Pearson's, Figure $3 \mathrm{H}$ ). The correlation between peak instantaneous rate and peak fluorescence was also significant but not high (0.51; Figure 3l). Other metrics, such as $\triangle F / F(t)$ time-integral and slope change had similar correlation values. Thus, G-CaMP imaged from PN somata appears to have low sensitivity for spiking activity, but to be reasonably correlated with sustained spiking at high firing rates. The poor kinetics and high threshold of the sensor at this expression level meant that computing a transfer function between G-CaMP signal and instantaneous firing rate was not practical.

\section{G-CaMP responses in glomeruli}

The response of any calcium sensor depends on the specifics of the compartment being imaged, e.g., the number and types of calcium channels present locally and the existence and nature of intracellular calcium stores (Yasuda et al., 2004). In insects as in most invertebrates, neuron somata-PNs, included-are unipolar; a primary neurite usually gives rise to "dendritic" and "axonal" compartments. The soma is thus not the integrative bottleneck that it is in most vertebrate neurons. In addition, PN glomerular neurites contain both pre- and post-synaptic specializations (Olsen et al., 2007; Shang et al., 2007; Wilson et al., 2004), and thus, likely have a high concentration of calcium channels. We investigated whether G-CaMP signals sampled from glomeruli are more sensitive than when they are measured from somata.

We compared G-CaMP signals from equally sized areas of the DL1 glomerulus and of the corresponding DL1 PN somata (NP3529-Gal4, UAS-GCaMP flies). Those regions were sampled successively in each experiment (three preparations) for direct comparison. Whereas baseline fluorescence was higher in the soma, response signals ( $\triangle F / F$ ) from the two regions were qualitatively similar and of similar peak amplitude. The main differences concerned response dynamics: response onset in the glomerulus preceded that in the soma by a long delay $(\mathrm{d} t=400 \pm 226 \mathrm{~ms}$ between crossings of $+2.5 \mathrm{SDs}$ of baseline; Figures $4 \mathrm{~A}-4 \mathrm{D}$ ) and response-onset slope was higher (i.e., rise time was faster) in the glomerulus. This may reflect differences between actual $[\mathrm{Ca} 2+]$ changes as well as differences in the sensitivity of G-CaMP in the two compartments. First, the glomerular response is the sum of G-CaMP signal from the dendrites of 2 DL1 PNs. Second, G-CaMP expression levels are lower in the glomerulus, reducing the buffering effect of the sensor and improving its observed kinetics (Yasuda et al., 2004). Third, dendritic compartments likely have a different distribution of voltage-dependent calcium channels and different endogenous calcium buffers than the soma (Baden and Hedwig, 2007; Single and Borst, 2002).

Next, we combined loose-patch recordings from DL1 PN somata and GCaMP imaging from the DL1 glomerulus. By tracking the neurites between somata and glomerulus, we could confirm the correspondence between the two recording sites ( $n=$ two preparations). We found that the faster kinetics of the G-CaMP glomerular responses indeed better represented electrical activity in the PNs (Figures $4 \mathrm{E}-4 \mathrm{H}$ ). Yet, glomerular G-CaMP 

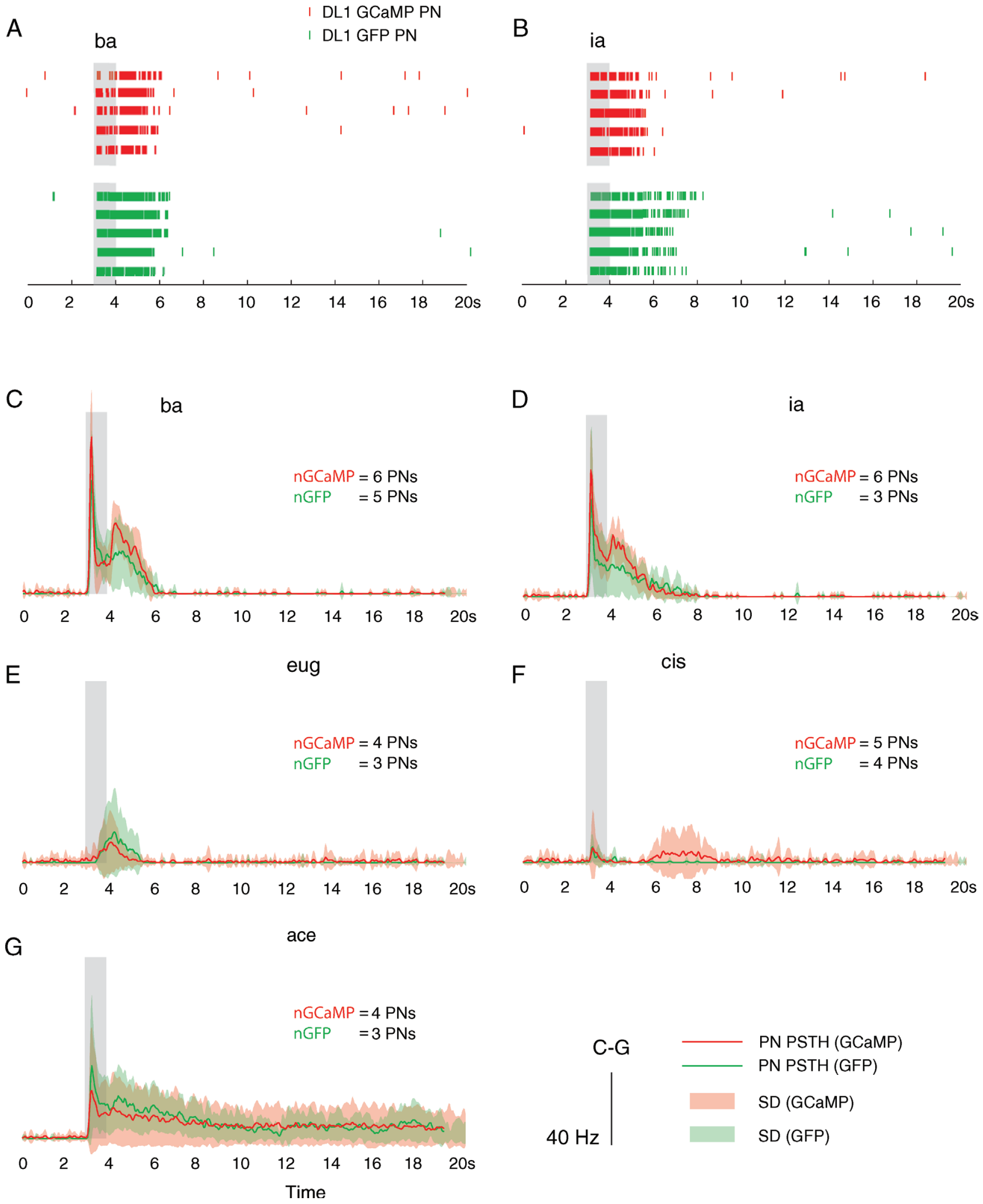

Figure 2. G-CaMP-expressing PNs (of the DL1 glomerulus) and their GFP-expressing counterparts do not show significantly different response patterns. (A-B) Sample responses of two PNs. The first five rows show spike rasters of single trial responses of a G-CaMP-expressing PN (NP3529-Gal4, UAS-GCaMP) fly, and the next five show responses of a GFP-expressing PN (NP3529-Gal4, UAS-eGFP) to ba and ia respectively. (C-G) Shown are mean PSTHs for DL1 PN: GFP (in green, with SD in light green) and G-CaMP (in red; SD in pink). PN spiking responses to different odors shown were smoothed (5 ms width Gaussian) before trial-averaging to produce PSTHs. G-CaMP PN responses were almost always within one SD of GFP PN responses (see stats in text). 
A

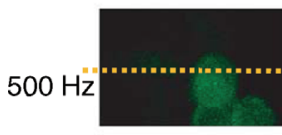

$\overline{5 \mu \mathrm{m}}$

B

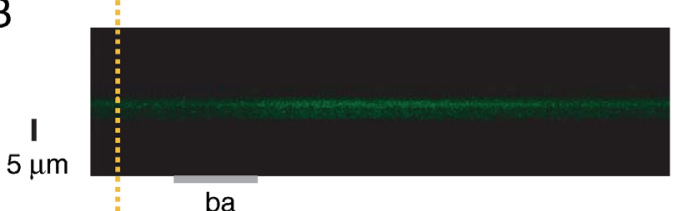

C

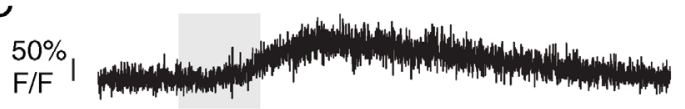

D

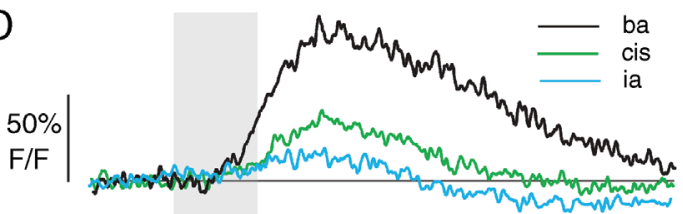

E

$10 \mathrm{pA}$

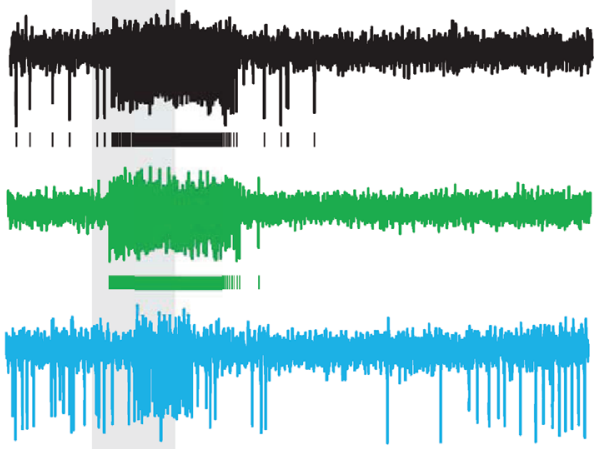

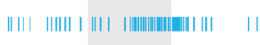

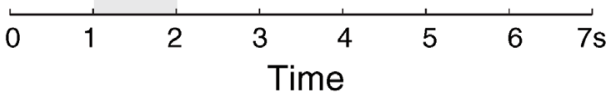

F

ba
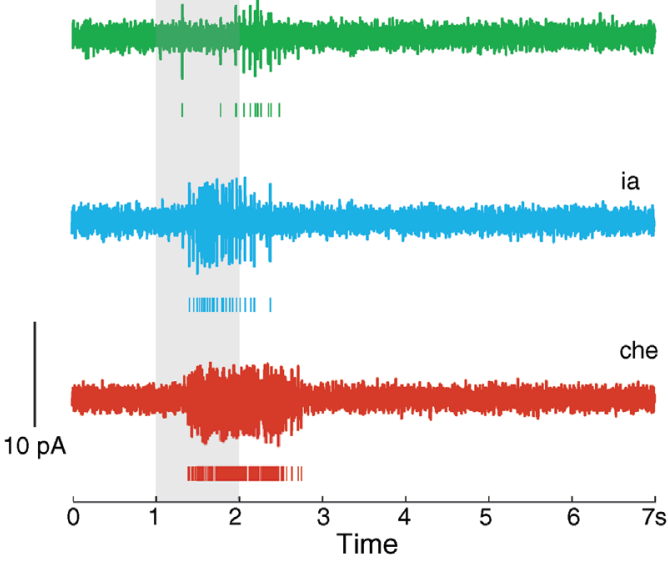

G
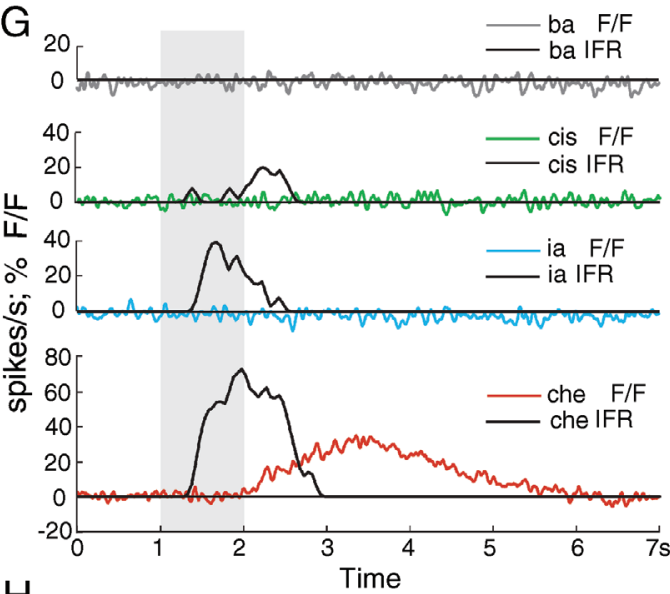

$\mathrm{H}$
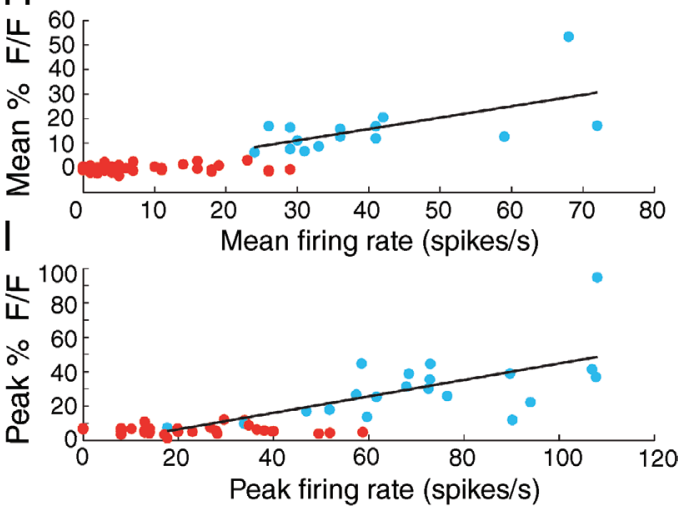

Figure 3. Simultaneous electrophysiology and 2-photon G-CaMP imaging of GH146-Gal4, UAS-GCaMP PNs. (A) Shows (from left) G-CaMP-positive PN in green channel, patch electrode in red (outline of approximate electrode position drawn by hand), merge. Yellow dashed line shows line chosen for line scanning in this experiment. (B) Results of line scan. Shown is the change in intensity levels as the odor is presented. (C) Raw and unfiltered single trial imaging signal $(\Delta F / F)$-based on integrating over the appropriate window in B-shows clear response to odor pulse, but with slow rise time. (D) Filtered G-CaMP signal shows responses to three odors (ba, cis, and ia). Imaging signal in response to ia is just barely detectable. (E) Simultaneously recorded electrophysiological signal showing PN responses to ba, cis, and ia. For each odor response, the first row shows raw signal, second row shows spikes detected. (F) Electrical recordings of odor responses of a PN from a different fly (also GH146-Gal4, UAS-GCaMP). (G) Results of simultaneous line scanning of the same PN's soma for the same odors. Shown is the change in fluorescence levels as the odor is presented. Also shown for comparison is the instantaneous firing rate based on simultaneous electrical recordings. G-CaMP signal shows high threshold of activation. Response to ia evoked greater than $40 \mathrm{~Hz}$ of activity, but did not evoke any G-CaMP signal. (H) Mean $\triangle F / F$ following odor presentation is partially correlated with the mean firing rate (during 1 second odor period) of various PNs in response to odor presentations. Red dots represent points that fall within 2.5SDs of baseline variation). Best-fit linear regression lines for significant points shown in black. Correlation value for trials with significant $\Delta F / F$ values: 0.61 . Pearson's $r^{2}$ is thus0 .38. (I) Peak $\Delta F / F$ following odor presentation is partially correlated with the peak firing rate (during 1 second odor period) of various PNs in response to odor presentations. Red dots represent points that fall within range of peak baseline variation). Best-fit linear regression lines for significant points shown in black. Correlation value for trials with significant $\Delta F / F$ values: 0.51 . Pearson's $r^{2}$ is thus 0.26 .

6 

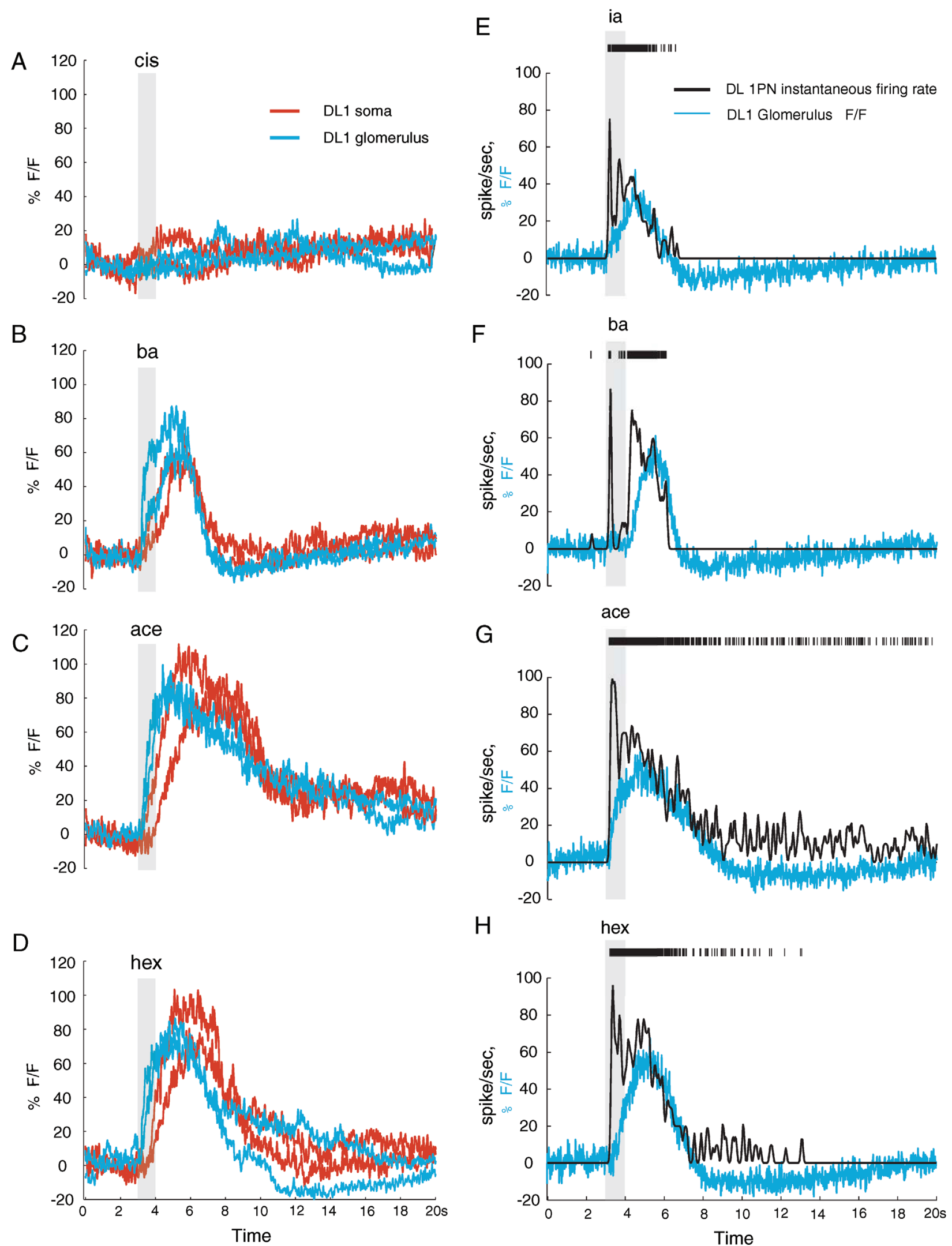

Figure 4. G-CaMP signal can be dependent on location imaged. (A-D) Glomerular G-CaMP signal versus somatic G-CaMP signal for the same DL1 PN (NP3529-Gal4, UAS-GCaMP fly) shows different kinetics. Shown are two trials each of somatic and glomerular line scans (order: soma-glomerulus-somaglomerulus, all odors tested at each step). Glomerular signal precedes somatic signal by $400 \pm 226 \mathrm{~ms}$ as measured by odor response signal exceeding $2.5 \mathrm{SD}$ s of baseline. (E-H) Comparison of instantaneous firing rate based on somatic electrical recordings and glomerular delta F/F. Although the G-CaMP signals show improved kinetics, they do not accurately capture the dynamics of the response, failing to correctly capture short bursts of intense electrical activity as high as $80 \mathrm{~Hz}$. Mean correlation between G-CaMP signal and instantaneous firing rate for these experiments $=0.71 \pm 0.04$. 
responses still failed to capture the dynamics of the PN response adequately, to the extent that even high-instantaneous-firing-rates segments of a response could go undetected if those were not sustained (Figures $4 \mathrm{E}-4 \mathrm{H})$. Over all trials in these experiments, G-CaMP failed to report even sustained ( $>1$ second) activity if it was below $30 \mathrm{sp} /$ second or to capture fast modulations of instantaneous firing rate (up to $80 \mathrm{sp} / \mathrm{second}$ ), even when those occurred on top of on-going plateaus of activity. For responses with significant $(p<0.001)$ correlation between instantaneous firing rate and G-CaMP signal during the odor response, the average correlation was $0.51 \pm 0.24$. (For the experiments shown in Figures $4 \mathrm{E}$ $4 \mathrm{H}$, the mean correlation was $0.71 \pm 0.04$.) Note also that fluorescence response kinetics between the two fly lines were different-the effect, presumably, of unequal promotor strengths and consequently, levels of G-CaMP expression-lower levels producing faster kinetics (Reiff et al., 2005; Yasuda et al., 2004).

\section{DISCUSSION}

We combined in vivo loose-patch recordings and two-photon calcium imaging with neurons in the Drosophila AL, to evaluate the correspondence between stimulus-evoked spike output and G-CaMP signals from soma or dendrites. This approach should be generally useful to evaluate genetically encoded sensors, activators, and repressors of neural activity, and to refine the study of neurons first identified genetically in a larger neuron population.

While stable for many minutes, loose-patch recordings usually deteriorated enough over 30 minute to render the recorded data unusable after this. The principal underlying reasons were electrode-clogging, large increase in seal resistance, and accidental break-ins. These experiments thus needed to be performed relatively quickly. We found, however, that the expression of the calcium indicator G-CaMP by DL1 PNs had no more effect on the electrophysiological responses of these PNs than GFP expression. This suggests that G-CaMP per se, at the genetic dosages tested here, may have no major deleterious effect on PN spiking activity.

\section{Does G-CaMP represent spiking activity faithfully?}

G-CaMP has become a widely used sensor of neural activity in Drosophila (Marella et al., 2006; Suh et al., 2004; Wang et al., 2003; Wang et al., 2004; Yu et al., 2006). Some studies have interpreted sparse G-CaMP 1.3 signals as indicative of sparse neuronal responses in the regions targeted for imaging (Marella et al., 2006; Wang et al., 2003; Wang et al., 2004). Our results, however, indicate that G-CaMP 1.3 is a low-sensitivity indicator of spiking activity, able to miss even high instantaneous discharge rates if activity is not sustained. These findings are broadly consistent with observations from hippocampal neurons of cultured brain slices (Pologruto et al., 2004) and Drosophila larval neuromuscular junction (Reiff et al., 2005). Also, G-CaMP responses show different kinetics and sensitivity when examined in the soma and dendritic compartments, or in different fly lines. This variability might arise because of spatial differences in expression levels, which is known to have effects on kinetics and $\mathrm{S} / \mathrm{N}$ ratio of calcium sensors (Reiff et al., 2005; Yasuda et al., 2004), in baseline calcium levels, and in the local calcium environment (channels, intracellular stores, and buffers). If generally applicable, our findings suggest that conclusions about neural coding based on G-CaMP imaging should be drawn with caution. In addition to general calibration tests in systems more amenable to electrophysiology (e.g., cultured slices; (Pologruto et al., 2004); Drosophila larval neuromuscular junction; (Reiff et al., 2005), tests should be carried out in vivo in the specific systems of interest. The mismatch we observe also provides one possible explanation for the discrepancy between electrophysiological (Olsen et al., 2007; Wilson et al., 2004) and G-CaMP imaging (Root et al., 2007; Wang et al., 2003) measurements of PN tuning. A high threshold of G-CaMP activation would of course artificially sparsen apparent odor-evoked activity. There may be yet other reasons for the different results: differences in the preparation (whole fly with intact antennae and palps instead of iso- lated antennae-brain preparations), saline, odor concentrations, and odor delivery systems used (for example, odors delivered in air rather than to antennae immersed in saline (Root et al., 2007)), holding potentials and consequences of whole-cell recordings (e.g., dialysis) affecting the odor responses seen. More broadly, our findings suggest that, at least at the expression level tested, G-CaMP appears not to have the combination of high S/N ratio, low activation threshold, and linear (or at least quantifiable) relationship to spiking activity that would make it a suitable substitute for electrophysiological approaches. The development of new and improved sensors (Mank et al., 2006; Reiff et al., 2005), however, provides hope.

The technique of two-photon targeted loose-patch recordings provides a useful tool to calibrate sensors in vivo in neurons of interest in the fly brain. Our results show that calibration is crucial for the correct interpretation of signals from such reporters. The method should also be more broadly useful in studies where imaging can be used to identify loci of interest that can then be targeted for further and more sensitive exploration with an electrode.

\section{CONFLICT OF INTEREST STATEMENT}

The authors declare that the research was conducted in the absence of any commercial or financial relationships that could be construed as a potential conflict of interest.

\section{ACKNOWLEDGEMENTS}

We would like to thank Glenn Turner for his generous advice throughout the project, and Rachel Wilson for her helpful tutoring on the fly preparation. We would also like to thank Laurent Moreaux for his assistance with twophoton microscopy and for technical advice, Mala Murthy for useful input and NP3529-Gal4, UAS-eGFP flies, Stijn Cassenaer, Benjamin Rubin and Anusha Narayan, and other members of the Laurent lab for useful technical discussions throughout the course of the project, and Suzuko Yorozu for advice on fly husbandry. Thanks also to Allan Wong for useful discussions.

Work supported by grants from the National Institute for Deafness and Communication Disorders, the National Science Foundation and the Gimbel Fund to GL, and a pre-doctoral fellowship from the Sloan-Swartz Center for Theoretical Neurobiology at Caltech to V. J.

\section{REFERENCES}

Baden, T., and Hedwig, B. (2007). Neurite-specific Ca2+ dynamics underlying sound processing in an auditory interneurone. Dev. Neurobiol. 67, 68-80.

Benzer, S. (1967). Behavioral mutants of Drosophila isolated by countercurrent distribution. Proc. Natl. Acad. Sci. USA 58, 1112-1119.

Brand, A. H., and Perrimon, N. (1993). Targeted gene expression as a means of altering cell fates and generating dominant phenotypes. Development 118, 401-415.

Cohen, L. B., and Salzberg, B. M. (1978). Optical measurement of membrane potential. Rev. Physiol. Biochem. Pharmacol. 83, 35-88.

Cohen, L. B., Salzberg, B. M., and Grinvald, A. (1978). Optical methods for monitoring neuron activity. Annu. Rev. Neurosci. 1, 171-182.

Denk, W., Strickler, J. H., and Webb, W. W. (1990). Two-photon laser scanning fluorescence microscopy. Science 248, 73-76.

Fiala, A., Spall, T., Diegelmann, S., Eisermann, B., Sachse, S., Devaud, J. M., Buchner, E., and Galizia, C. G. (2002). Genetically expressed cameleon in Drosophila melanogaster is used to visualize olfactory information in projection neurons. Curr. Biol. 12, 1877-1884.

Grinvald, A., and Hildesheim, R. (2004). VSDI: a new era in functional imaging of cortical dynamics. Nat. Rev. Neurosci. 5, 874-885.

Grynkiewicz, G. Poenie, M. and Tsien, R. Y (1985). A new generation of Ca2+ indicators with greatly improved fluorescence properties. J. Biol. Chem. 260, 3440-3450.

Helmchen, F., Imoto, K., and Sakmann, B. (1996). Ca2+ buffering and action potentialevoked $\mathrm{Ca} 2+$ signaling in dendrites of pyramidal neurons. Biophys. J. 70, 1069-1081.

Hotta, Y., and Benzer, S. (1970). Genetic Dissection of Drosophila Nervous System by Means of Mosaics. Proc. Natl. Acad. Sci. USA 67, 1156

Mank, M., Reiff, D. F., Heim, N., Friedrich, M. W., Borst, A., and Griesbeck, 0. (2006). A FRET-based calcium biosensor with fast signal kinetics and high fluorescence change. Biophys. J. 90, 1790-1796.

Marella, S., Fischler, W., Kong, P., Asgarian, S., Rueckert, E., and Scott, K. (2006). Imaging taste responses in the fly brain reveals a functional map of taste category and behavior. Neuron 49, 285-295.

Margrie, T. W., Meyer, A. H., Caputi, A., Monyer, H., Hasan, M. T., Schaefer, A. T., Denk, W., and Brecht, M. (2003). Targeted whole-cell recordings in the mammalian brain in vivo. Neuron $39,911-918$. 
Miyawaki, A., Llopis, J., Heim, R., McCaffery, J. M., Adams, J. A., Ikura, M., and Tsien, R. Y. (1997). Fluorescent indicators for $\mathrm{Ca} 2+$ based on green fluorescent proteins and calmodulin. Nature 388, 882-887.

Nakai, J., Ohkura, M., and Imoto, K. (2001). A high signal-to-noise $\mathrm{Ca}(2+)$ probe composed of a single green fluorescent protein. Nat. Biotechnol. 19, 137-141.

Ng, M., Roorda, R. D., Lima, S. Q., Zemelman, B. V., Morcillo, P., and Miesenbock, G. (2002). Transmission of olfactory information between three populations of neurons in the antennal lobe of the fly. Neuron 36, 463-474.

Olsen, S. R., Bhandawat, V., and Wilson, R. I. (2007). Excitatory interactions between olfactory processing channels in the Drosophila antennal lobe. Neuron 54, 89-103.

Persechini, A., Lynch, J. A., and Romoser, V. A. (1997). Novel fluorescent indicator proteins for monitoring free intracellular Ca2+. Cell Calcium 22, 209-216.

Pologruto, T. A., Yasuda, R., and Svoboda, K. (2004). Monitoring neural activity and [Ca2+] with genetically encoded Ca2+ indicators. J. Neurosci. 24, 9572-9579.

Quinn, W. G., Harris, W. A., and Benzer, S. (1974). Conditioned behavior in Drosophila melanogaster. Proc. Natl. Acad. Sci. USA 71, 708-712.

Reiff, D. F., Ihring, A., Guerrero, G., Isacoff, E. Y., Joesch, M., Nakai, J., and Borst, A. (2005). In vivo performance of genetically encoded indicators of neural activity in flies. J. Neurosci. 25, 4766-4778.

Root, C. M., Semmelhack, J. L., Wong, A. M., Flores, J., and Wang, J. W. (2007) Propagation of olfactory information in Drosophila. Proc. Natl. Acad. Sci. USA.

Sakmann, B., and Neher, E. (1984). Patch clamp techniques for studying ionic channels in excitable membranes. Annu. Rev. Physiol. 46, 455-472.

Schroll, C., Riemensperger, T., Bucher, D., Ehmer, J., Voller, T., Erbguth, K., Gerber, B. Hendel, T., Nagel, G., Buchner, E., and Fiala, A. (2006). Light-induced activation of distinct modulatory neurons triggers appetitive or aversive learning in Drosophila larvae. Curr. Biol. 16, 1741-1747.

Shang, Y., Claridge-Chang, A., Sjulson, L., Pypaert, M., and Miesenbock, G. (2007). Excitatory local circuits and their implications for olfactory processing in the fly antennal lobe. Cell 128, 601-612.

Single, S., and Borst, A. (2002). Different mechanisms of calcium entry within different dendritic compartments. J. Neurophysiol. 87, 1616-1624.

Smetters, D., Majewska, A., and Yuste, R. (1999). Detecting action potentials in neuronal populations with calcium imaging. Methods 18, 215-221.

Stosiek, C., Garaschuk, 0., Holthoff, K., and Konnerth, A. (2003). In vivo two-photon calcium imaging of neuronal networks. Proc. Natl. Acad. Sci. USA 100, 7319-7324.

Su, H., and O'Dowd, D. K. (2003). Fast synaptic currents in Drosophila mushroom body Kenyon cells are mediated by alpha-bungarotoxin-sensitive nicotinic acetylcholine receptors and picrotoxin-sensitive GABA receptors. J. Neurosci. 23 9246-9253.

Suh, G. S., Wong, A. M., Hergarden, A. C., Wang, J. W., Simon, A. F., Benzer, S., Axel, R., and Anderson, D. J. (2004). A single population of olfactory sensory neurons mediates an innate avoidance behaviour in Drosophila. Nature.

Svoboda, K., Denk, W., Kleinfeld, D., and Tank, D. W. (1997). In vivo dendritic calcium dynamics in neocortical pyramidal neurons. Nature 385, 161-165.

Tanaka, N. K., Awasaki, T., Shimada, T., and Ito, K. (2004). Integration of chemosensory pathways in the Drosophila second-order olfactory centers. Curr. Biol. 14, 449-457.

Tank, D. W., Sugimori, M., Connor, J. A., and Llinas, R. R. (1988). Spatially resolved calcium dynamics of mammalian Purkinje cells in cerebellar slice. Science 242, 773-777.

Taylor, A. L., Cottrell, G. W., Kleinfeld, D., and Kristan, W. B., Jr. (2003). Imaging reveals synaptic targets of a swim-terminating neuron in the leech CNS. J. Neurosci. 23, $11402-11410$

Wang, J. W., Wong, A. M., Flores, J., Vosshall, L. B., and Axel, R. (2003). Two-photon calcium imaging reveals an odor-evoked map of activity in the fly brain. Cell 112, 271-282.

Wang, Y., Guo, H. F., Pologruto, T. A., Hannan, F., Hakker, I., Svoboda, K., and Zhong, $Y$. (2004). Stereotyped odor-evoked activity in the mushroom body of Drosophila revealed by green fluorescent protein-based Ca2+ imaging. J. Neurosci. 24, $6507-6514$.

Wilson, R. I., and Laurent, G. (2005). Role of GABAergic inhibition in shaping odorevoked spatiotemporal patterns in the Drosophila antennal lobe. J. Neurosci. 25, 9069-9079.

Wilson, R. I., Turner, G. C., and Laurent, G. (2004). Transformation of olfactory representations in the Drosophila antennal lobe. Science 303, 366-370.

Yasuda, R., Nimchinsky, E. A., Scheuss, V., Pologruto, T. A., Oertner, T. G., Sabatini, B. L., and Svoboda, K. (2004). Imaging calcium concentration dynamics in small neuronal compartments. Sci STKE 2004, 15.

Yu, D., Akalal, D. B., and Davis, R. L. (2006). Drosophila alpha/beta mushroom body neurons form a branch-specific, long-term cellular memory trace after spaced olfactory conditioning. Neuron 52, 845-855.

Yu, D., Ponomarev, A., and Davis, R. L. (2004). Altered representation of the spatial code for odors after olfactory classical conditioning; memory trace formation by synaptic recruitment. Neuron 42, 437-449.

Yuste, R., and Katz, L. C. (1991). Control of postsynaptic Ca2+ influx in developing neocortex by excitatory and inhibitory neurotransmitters. Neuron 6, 333-344. 